\title{
Aktuelle Standards in der Behandlung des Pankreaskarzinoms
}

\author{
Uwe Pelzer, Hanno Riess
}

\section{Stand der Dinge}

Das Adenokarzinom des Pankreas ist eine der prognostisch ungünstigsten Tumorentitäten. Die Prognose ist für die Mehrzahl der Patienten primär infaust. Dies ist der Tatsache geschuldet, dass frühe Krankheitssymptome unspezifisch sind. Da auch ausreichend sensitive Laborparameter fehlen, ist die Verdachtsdiagnose „Pankreaskarzinom" freizügig zu stellen und in die differenzialdiagnostische Abklärung von Allgemeinsymptomen oder unklaren persistierenden Oberbauchbeschwerden mit einzubeziehen [1-3].

Symptomorientierte Therapie I Gleichrangig neben der tumorspezifischen Behandlung sollte von Diagnose an die symptomorientierte Therapie stehen, um Schmerz und Kachexie frühzeitig zu behandeln, einen fortschreitenden Gewichtsverlust mit Verschlechterung des Allgemeinzustandes zu verhindern und krankheitsspezifische Komplikationen bereits präventiv zu therapieren $[4,5]$. Geeignete Maßnahmen, wie Sicherung des Galleabflusses und der Magen-Darm-Passage oder die medikamentöse Thromboembolieprophylaxe, lassen Komplikationen wie Verschlussikterus, Cholangitiden, Magenausgangs- bzw. Duodenalobstruktionen sowie Thromboembolien vermeiden [6, 7]. Bei mehr als zwei Drittel der Patienten liegt bei Diagnosestellung ein lokal fortgeschrittenes inoperables oder metastasiertes Stadium vor; in der Mehrzahl der Fälle ist hier eine palliative Chemotherapie indiziert. Vor Einleitung einer spezifischen Therapie soll eine zytologische oder histologische Sicherung der Diagnose „Adenokarzinom“ regelhaft erfolgen ( Abb. 1) [1].

\section{Frühe Stadien: Lokal resektable, nicht metastasierte Tumore}

Tumorresektion | Die chirurgische Resektion bietet aktuell die einzig kurative Prognose. Ziel ist eine komplette Tumorentfernung im Gesunden (R0) mit Resektion von regionären Lymphknoten (mindestens 10!). Eine erweiterte Lymphadenektomie wird nicht empfohlen. Ein Update der S3Leitlinien zum Pankreaskarzinom hat das Konzept des zirkumferenziellen Resektionsrandes (CRM) analog zum Rektumkarzinom übernommen [1]:

- R1-Stadium: mikroskopisch nachweisbare Tumorzellen am definierten Resektionsrand

- R0-,wide“-Stadium: Abstand des Tumors zum Resektionsrand $\geq 1 \mathrm{~mm}$

\section{Was ist neu?}

- Frühe Stadien: Nach Diagnose sollte eine zügige Operation mit dem Ziel einer R0 intendierten Resektion erfolgen. Die Aufarbeitung des Resektates erfolgt nach aktualisierten standardisierten Prozeduren. Nach kurativ intendierter Operation zeigt eine 6-monatige adjuvante Chemotherapie eine relevante Verbesserung der Kurationsrate. Ergebnisse intensiverer postoperativer Therapiestrategien liegen bisher noch nicht vor.

- Lokal fortgeschrittene Stadien: Die lokale Strahlentherapie der Primariusregion zeigt keinen Vorteil gegenüber einer palliativen systemischen Therapie. Aufgrund der steigenden Effizienz kombinierter Chemotherapien werden neoadjuvante/Konversions-Strategien unter Einschluss der Strahlentherapie in kontrollierten Studien geprüft.

- Palliative Systemtherapie: Bei inoperablen oder metastasierten Stadien verlängert eine Chemotherapie das Überleben signifikant. Dabei stehen mehrere nachgewiesenermaßen wirksame Erstlinientherapien mit unterschiedlichem Toxizitätsprofil zur Verfügung, so dass dem Allgemeinzustand der Patienten Rechnung getragen werden kann.

- Zweitlinientherapie: Eine Zweitlinientherapie sollte Patienten mit ausreichendem Allgemeinzustand in Abhängigkeit von der Art der Erstlinientherapie angeboten werden. Es zeigt sich ein Überlebensvorteil gegenüber der alleinigen besten supportiven Behandlung.

- CRM-positiv, bzw. R0-,narrow“: Abstand des Tumors zum Resektionsrand $<1 \mathrm{~mm}$

Die präoperative Bildgebung lässt oft keine eindeutige Festlegung einer Resektabilität zu, meist aufgrund des fraglichen Ausmaßes der Infiltration der beteiligten Arterien. International setzt sich zunehmend auch eine Definition für borderline-resektable lokal fortgeschrittene Pankreaskarzinome durch. Hier ist es sinnvoll, bezüglich der Operabilität eine Zweitmeinung in einem „High Volume“-Referenzzentrum für Pankreaschirurgie einzuholen.

Adjuvante Chemotherapie I Trotz einer primär erfolgreichen R0-Resektion ist die Prognose ernst: Ein Krankheitsrezidiv innerhalb der nächsten 2 Jahre ist sehr wahrscheinlich. Eine adjuvante Chemotherapie mit Gemcitabin kann die Prognose signifikant verbessern. Bei Unverträglichkeit von Gemcitabin ist das 5-FU-Bolusschema als Alternative zu empfehlen [14, 15]. Für die Wirksamkeit sind die 6-monatige Dauer sowie die Dosisintensität, weniger ein früher Therapiebeginn wesentlich. Vor Beginn ist eine aussagekräftige Schnittbildgebung (z. B. CT-Thorax / Abdomen) zu fordern, um ein frühes postoperatives Rezidiv auszuschließen.

Aufbauend auf der Verdoppelung des 5-Jahresüberlebens durch die adjuvante Chemotherapie 


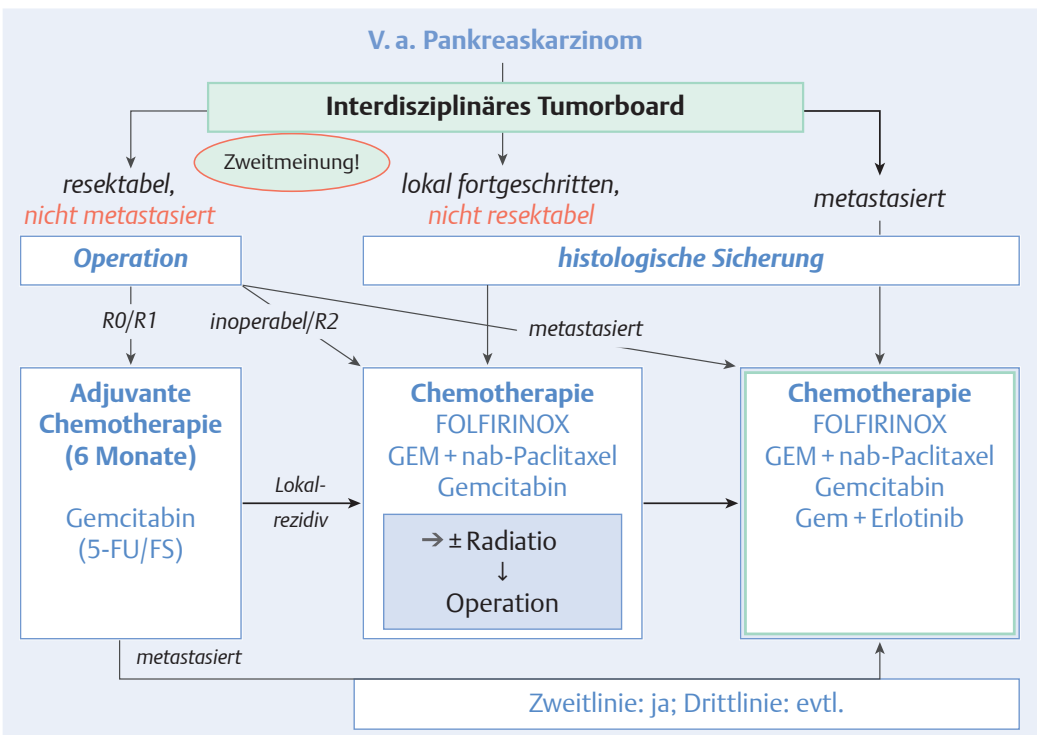

Abb. 1 Behandlungsalgorithmus für Patienten mit Adenokarzinom des Pankreas.
Studienkonzepte zur Verfügung stehen, die diese Fragestellung sinnvoll untersuchen.

\section{Palliative Systemtherapie}

Trotz der bei fortgeschrittener Erkrankung infausten Prognose ist kein therapeutischer Nihilismus angezeigt. Eine individualisierte Therapie kann krankheitsbedingte Symptome verzögern und das Überleben verlängern.

Erstlinientherapie I Prospektive randomisierte Studien der letzten zwei Dekaden, welche die Gemcitabin-Standardtherapie mit Chemotherapie-Kombinationen, zielgerichteten Therapeutika oder deren Kombinationen mit Gemcitabin untersucht haben, sind ohne wesentlichen Behandlungsfortschritt geblieben. In den letzten 5 Jahren wurde jedoch zweifelsfrei nachgewiesen, dass intensivere Chemotherapiestrategien bei dafür geeigneten Patienten zu einem signifikanten, und klinisch relevanten Überlebensvorteil bei erhaltener bzw. verbesserter Lebensqualität führen ( $\bullet$ Tab. 1).

Nach einer ersten positiven Studie mit Gemcitabin + Erlotinib [8], ergab sich für die Kombination aus Nab-Paclitaxel + Gemcitabin ein verbessertes medianes Gesamtüberleben von 6,7 auf 8,2 Monate. Dabei traten vermehrt Hämatotoxizität, Fatigue und reversible sensorische Polyneuropathien auf [9]. Die Gemcitabin-freie Kombination FOLFIRINOX zeigte im Vergleich zur GemcitabinMonotherapie eine bessere mediane Überlebenszeit (6,8 vs. 11,1 Monate) mit vermehrter Hämatotoxizität, Diarrhoe und Oxaliplatin-assoziierter (meist nur partieller oder nicht reversibler) Polyneuropathie [10]. Diese beiden neuen Therapieprotokolle sind geeignet für Patienten in gutem bis sehr gutem Allgemeinzustand, einem geringen Risiko für Cholangitiden und anderen infektiösen, Neutropenie-bedingten Komplikationen.

Lokal inoperable, nicht metastasierte Tumore Patienten mit lokal fortgeschrittenen, irresektablen, aber nicht metastasierten Pankreaskarzinomen unterscheiden sich im spontanen Verlauf ebenso wie unter Therapie von Patienten mit metastasierten Tumorstadien. Untersuchungen geben Hinweise darauf, dass die molekularen Veränderungen heterogen sind. Der generelle Einsatz der Strahlentherapie muss als weitgehend unwirksam eingeordnet werden; nicht zuletzt weil bei vielen dieser Patienten bereits eine subklinisch metastasierte Situation vorliegt. Insbesondere nach gutem Ansprechen auf die primär durchzuführende systemische Therapie wird dennoch und mit nachvollziehbarer Rationale die Rolle der Strahlentherapie in multimodalen Therapiekonzepten, insbesondere zur neoadjuvanten Therapie bei primär nicht, oder nur borderlineresektablem Pankreaskarzinom geprüft. Der Einsatz derartiger Therapiekonzepte im Rahmen der Versorgungsmedizin muss kritisch hinterfragt werden, zudem auch in Deutschland mehrere
Aktuelle Daten aus zwei deutschen Patientenregistern (ONKOReg, TPK) zeigen, dass diese evidenzbasierten neuen Therapiestrategien in die Versorgungswirklichkeit integriert werden und die Effektivitätsdaten der Zulassungsstudien bestätigen. So wurden bei etwa $60 \%$ der Patienten FOLFIRINOX ( $20 \%)$ bzw. Nab-Paclitaxel $(\sim 40 \%)$ und in etwa $35 \%$ die meist besser verträgliche Gemcitabin-Monotherapie durchgeführt [11, 12].

Dauer der Erstlinientherapie | Bei gutem bzw. befriedigendem Therapieansprechen und vertretbarer Toxizität nach mehrmonatiger Therapie besteht kein Konsens über die Intensität und Dauer einer Therapiefortführung. Die unter FOLFIRINOX regelhaft auftretende Oxaliplatin-assoziierte Polyneuropathie limitiert die Dauer in aller Regel auf 6 bis 12 Anwendungen (d.h. 3-6 Monate). Ob 


\begin{tabular}{|c|c|c|c|c|c|c|}
\hline & \multicolumn{2}{|c|}{ Conroy [10] } & \multicolumn{2}{|c|}{ Von Hoff [9] } & \multicolumn{2}{|l|}{ Moore [8] } \\
\hline Medianes Alter & \multicolumn{2}{|c|}{61 [25-75] Jahre } & \multicolumn{2}{|c|}{63 [27-88] Jahre } & \multicolumn{2}{|l|}{64 [36-92] } \\
\hline Performance-Status & \multicolumn{2}{|c|}{ 0/1 (ECOG) } & \multicolumn{2}{|c|}{$\geq 70$ (KPS) } & \multicolumn{2}{|l|}{ 0/1/2 (ECOG) } \\
\hline Bilirubin & \multicolumn{2}{|c|}{$\leq 1,5 \mathrm{mg} / \mathrm{dl}$} & \multicolumn{2}{|c|}{$\leq 1 \mathrm{mg} / \mathrm{dl}$} & \multicolumn{2}{|l|}{$<2 x U L N$} \\
\hline DHC-Stent & \multicolumn{2}{|l|}{$16 \%$} & \multicolumn{2}{|l|}{$17 \%$} & \multicolumn{2}{|l|}{ k.a. } \\
\hline Chemotherapie & Gem & FOLFIRINOX & Gem & Nab-Paclitaxel + Gem & Gem + Placebo & Gem + Erlotinib \\
\hline Dosisintensität (\%) & $100 \%$ & $82 \%, 81 \%, 78 \%$ & $85 \%$ & $81 \%, 75 \%$ & k. A. & k. A. \\
\hline Ansprechrate & $9,4 \%$ & $31,6 \%$ & $8 \%$ & $29 \%$ & $8 \%$ & $8,6 \%$ \\
\hline mPFS (Monate) & 3,3 & 6,4 & 3,7 & 5,5 & 3,6 & 3,8 \\
\hline Hazard Ratio (95\%-KI) & \multicolumn{2}{|c|}{$0,47(0,37-0,59)$} & \multicolumn{2}{|c|}{$0,69(0,58-0,82)$} & \multicolumn{2}{|c|}{$0,77(0,64-0,92)$} \\
\hline mOS (Monate) & 6,8 & 11,1 & 6,7 & 8,5 & 5,9 & 6,2 \\
\hline Hazard Ratio (95\%-KI) & \multicolumn{2}{|c|}{$0,57(0,45-0,73)$} & \multicolumn{2}{|c|}{$0,72(0,62-0,84)$} & \multicolumn{2}{|c|}{$0,82(0,69-0,99)$} \\
\hline Neutropenie $\geq 3^{\circ}$ & $19 \%$ & $46 \%$ & $27 \%$ & $38 \%$ & $29 \%$ & $24 \%$ \\
\hline Polyneuropathie $\geq 3^{\circ}$ & $0 \%$ & $9 \%$ & $<1 \%$ & $17 \%$ & k.a & k.a. \\
\hline Fatigue $\geq 3^{\circ}$ & $14,2 \%$ & $23,2 \%$ & $7 \%$ & $17 \%$ & $15 \%$ & $15 \%$ \\
\hline Diarrhoe $\geq 3^{\circ}$ & $1 \%$ & $12 \%$ & $1 \%$ & $6 \%$ & $2 \%$ & $6 \%$ \\
\hline
\end{tabular}

ECOG = Eastern Cooperative Oncology Group; KPS = Karnofsky Performance Status; DHC= Ductus hepaticus communis; Gem = Gemcitabin; $\mathrm{mPFS}=$ medianes progressionsfreies Überleben; $\mathrm{mOS}$ = medianes Gesamtüberleben.

in dieser Situation - Therapieansprechen bei vertretbarer Gesamttoxizität, aber klinisch relevant werdender Polyneuropathie - die Therapie insgesamt pausiert oder auf ein reduziertes FOLFIRISchema umgestellt werden sollte, ist nicht ausreichend geklärt. Wir versuchen in individueller Abstimmung mit dem Patienten, dem Therapieziel und den Parametern des Tumorverhaltens (tumorassoziierte Symptome, CA-19-9, Staging) eine individuelle Entscheidung zu treffen. Bei der Mehrzahl der von uns betreuten Patienten wird die Chemotherapie ohne Oxaliplatin bis zum Progress fortgeführt und das Therapieintervall mitunter von 14 auf 21 Tage gestreckt. Die bei NabPaclitaxel/Gemcitabin beobachteten Polyneuropathien sind beim Pausieren von Nab-Paclitaxel in aller Regel innerhalb weniger Wochen weitestgehend reversibel. Daher wird hier zunächst eine Fortführung als Gemcitabin-Monotherapie und abhängig vom weiteren Tumorverlauf eine intensivierende Hinzunahme von Nab-Paclitaxel nach Abklingen der Polyneuropathie von uns empfohlen.

Die bei intensiveren Therapieprotokollen (FOLFIRINOX, Nab-Paclitaxel/Gemcitabin) beobachtete Tumoransprechrate von über 20\% (31,6\% bzw. $29 \%$ ) macht bei symptomatischen Patienten eine rasche und längerfristige Symptomverbesserung wahrscheinlich. Darüber hinaus lässt sich bei einigen Patienten mit gutem Therapieansprechen eine sekundäre Operabilität erhoffen.

\section{Klinische Relevanz}

Zur palliativen Erstlinienstrategie stehen mehrere evidenzbasierte Therapieprotokolle zur Verfügung, die im Rahmen einer individuellen Nutzen- / Risikoabwägung in Betracht gezogen und mit den Patienten - ebenso wie die offenen Studienkonzepte - diskutiert werden sollten.

\section{Zweitlinientherapie}

Für Patienten mit progredienter Erkrankung gibt es mehrere Optionen einer Zweitlinientherapie. Bei der Wahl des Therapieschemas sind der Allgemeinzustand, die tumorassoziierten Symptome sowie die Tolerabilität/ Toxizität der Erstlinientherapie zu berücksichtigen. Für die neueren, intensiveren Kombinations-Erstlinientherapien gibt es keine ausreichend abgesicherte Studienlage zur optimalen Zweitlinientherapie. Das von uns etablierte OFF-Protokoll aus Oxaliplatin und 5-FU-Folinsäure hat sich jedoch als wirksam nach Gemcitabin-Monotherapie erwiesen [13] und im klinischen Alltag bewährt [11, 12]:

- medianes Überleben im Vergleich zu „Best Supportive Care“ 4,8 vs. 2,3 Monate (HR 0,45; $\mathrm{p}=0,008)$;

- medianes Überleben 5,9 vs. 3,3 Monate (HR 0,$66 ; p=0,01$ ) im Vergleich zu einer Chemotherapie aus 5-FU und Folinsäure.
Tab. 1 Studienlage zu den verfügbaren evidenzbasierten palliativen Chemotherapien. 


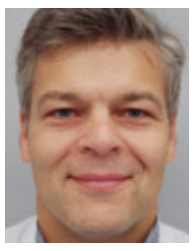

Priv. Doz. Dr. med. Uwe Pelzer

ist Facharzt und Arbeitsgruppenleiter an der Medizinischen Klinik m. S. Hämatologie, Onkologie und Tumorimmunologie, Universitätsmedizin Berlin - Charité. uwe.pelzer@charite.de

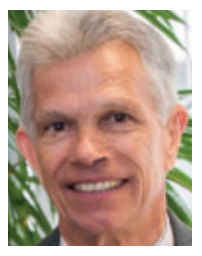

Univ.-Prof. Dr. med. Hanno Ries

ist stellvertretender Direktor an der Med. Klinik m.S. Hämatologie, Onkologie und Tumorimmunologie und Leiter der Internistischen Onkologie, Universitätsmedizin Berlin - Charité. hanno.riess@charite.de

\section{Interessenkonflikt} UP und HR haben erhalten: Beratungs-/ Vortragshonorare oder Kongressreiseunterstützung von Amgen, Baxalta, Celgene, Bayer, Lilly, BBraun-Travacare und Roche.

DOI 10.1055/s-0041-107888 Dtsch Med Wochenschr 2015; 140: 1813-1816 (c) Georg Thieme Verlag KG . Stuttgart $\cdot$ New York . ISSN 0012-0472
Die im Vergleich zur FOLFOX-Therapie (Folinsäure, 5-FU, Oxaliplatin; Schema beim kolorektalen Karzinom) reduzierte Therapieintensität ist für die Therapiedurchführbarkeit sowie die Lebensqualität von wesentlicher Bedeutung. Zukünftig könnte das nanoliposomale Irinotecan in Kombination mit 5-FU-Folinsäure eine Alternative sein. In einer Phase-III-Studie verbesserte sich nach Gemcitabin-Vortherapie das mediane Gesamtüberleben im Vergleich zu 5-FU-Folinsäure signifikant (6,1 vs. 4,2 Monaten; HR 0,67; p=0,012).

Nach FOLFIRINOX kann in Abhängigkeit von der Therapiefähigkeit eine Kombination von Gemcitabin + Nab-Paclitaxel oder eine Gemcitabin-Monotherapie in Betracht gezogen werden. Nach Nab-Paclitaxel + Gemcitabin wird den Patienten eine intensive Therapie wie FOLFIRINOX in aller Regel nicht zugemutet werden können; das OFFSchema ist hier oftmals durchführbar und erwägenswert.

Nachdem bereits die den Empfehlung einer Zweitlinientherapie zugrundeliegenden Evidenzen deutlich limitiert sind, ergibt sich entsprechend keine studiengemäß abgesicherte Empfehlung für eine konkrete Drittlinientherapie. Sie wird jedoch bei $5-10 \%$ der Patienten durchgeführt $[11,12]$. Bei ausreichendem Allgemeinzustand sollte den Patienten möglichst die Teilnahme an Phase-I- oder Phase-II-Studienkonzepten eröffnet werden.

\section{Klinische Relevanz \\ Nach einer Gemcitabintherapie hat sich das OFF-Schema etabliert. Bei Krankheitsprogres- sion unter einer FOLFIRINOX-Behandlung kommt eine Gemcitabin-Monotherapie oder Gemcitabin + Nab-Paclitaxel in Betracht.}

Individualisierte Therapie im fortgeschrittenen Tumorstadium | Der unter Therapie individuell unterschiedliche Krankheitsverlauf eröffnet für die selektierten Patienten diverse Behandlungsoptionen. Dies betrifft den Einsatz der Strahlentherapie bei stabilen Patienten mit fortbestehender lokaler inoperabler Erkrankung ohne Metastasierung, den Einsatz von chirurgisch Resektionsverfahren / Brachytherapie bei Oligometastasierung sowie die selektive interstitielle Radiotherapie bei führend diffuser Lebermetastasierung. Um mit Patienten die in Betracht kommenden - in dieser Tumorentität nicht evidenzgesicherten - Therapieoptionen fundiert diskutieren zu können, ist die Evaluation des individuellen Krankheitsverlaufs im interdisziplinären Tumorboard mit Kollegen sinnvoll und wünschenswert, die über praktische Erfahrung mit sämtlichen z.T. konkurrierenden Verfahren verfügen - eine großzügige Zweitmeinungsvorstellung in den wenigen großen deutschen Therapiezentren ist daher sinnvoll.

\section{Klinische Relevanz}

Je nach Krankheitsverlauf bieten sich verschiedene Therapieoptionen an. Sie sollten im Tumorboard besprochen und individuell mit dem Patienten abgestimmt werden.

\section{Literatur}

1 Seufferlein T, Porzner M, Becker T et al. S3-Leitlinie zum exokrinen Pankreaskarzinom. Z Gastroenterol 2013; 51: 1395-1440.

2 Pelzer U, Sinn M, Stieler ], Riess H. [Multimodal treatment of pancreatic cancer]. Internist (Berl) 2014; 55: 31-36.

3 Seufferlein T, Porzner M, Heinemann V et al. Ductal pancreatic adenocarcinoma. Dtsch Arztebl Int 2014; 111: 396-402

4 Pelzer U, Arnold D, Gövercin M et al. Parenteral nutrition support for patients with pancreatic cancer. Results of a phase II study. BMC Cancer 2010; 10: 86

5 Capodano G. [Supportive care in pancreatic cancer]. Rev Prat 2015; 65: 390-394.

6 Pelzer U, Opitz B, Deutschinoff G et al. Efficacy of Prophylactic Low-Molecular Weight Heparin for Ambulatory Patients With Advanced Pancreatic Cancer: Outcomes From the CONKO-004 Trial. J Clin Oncol 2015; 33: 2028-2034

7 Feisthammel ], Mössner J, Hoffmeister A. Palliative Endoscopic Treatment Options in Malignancies of the Biliopancreatic System. Viszeralmedizin 2014; 30: 238-243.

8 Moore MJ, Goldstein D et al. Erlotinib plus gemcitabine compared with gemcitabine alone in patients with advanced pancreatic cancer: a phase III trial of the National Cancer Institute of Canada Clinical Trials Group. J Clin Oncol 2007; 25: 1960-1966

9 Von Hoff DD, Ervin T, Arena FP et al. Increased survival in pancreatic cancer with nab-paclitaxel plus gemcitabine. N Engl J Med 2013; 369: 1691-1703.

10 Conroy T, Desseigne F, Ychou M et al. FOLFIRINOX versus gemcitabine for metastatic pancreatic cancer. N Engl J Med 2011; 364: 1817-1825

11 Weber K, Schlichting A, Tessen HW. Das Pankreaskarzinom - Versorgungsforschung und Versorgungsrealität in onkologischen Praxen. DGHO 2015

12 Hegewisch-Becker S, Hofheinz RD, Wolf T et al. Progressionsfreies und Gesamtüberleben von Patienten mit Pankreaskarzinom in Deutschland erste Outcome-Daten aus dem klinischen Tumorregister Pankreaskarzinom (TPK). DGHO 2015

13 Oettle H, Riess H, Stieler JM et al. Second-line oxaliplatin, folinic acid, and fluorouracil versus folinic acid and fluorouracil alone for gemcitabine-refractory pancreatic cancer: outcomes from the CONKO-003 trial. J Clin Oncol 2014; 32: 2423-2429

14 Neoptolemos JP, Stocken DD, Bassi C et al. Adjuvant chemotherapy with fluorouracil plus folinic acid vs gemcitabine following pancreatic cancer resection: a randomized controlled trial.JAMA 2010; 304: 1073-1081

15 Oettle H, Neuhaus P, Hochhaus A et al. Adjuvant chemotherapy with gemcitabine and long-term outcomes among patients with resected pancreatic cancer: the CONKO-001 randomized trial. JAMA 2013; 310: 1473-1481 